\title{
Chaos and Ergodicity in Extended Quantum Systems with Noisy Driving
}

\author{
Pavel Kos $\odot,{ }^{1}$ Bruno Bertini $\odot,{ }^{1,2}$ and Tomaž Prosen $\odot^{1}$ \\ ${ }^{1}$ Department of Physics, Faculty of Mathematics and Physics, University of Ljubljana, \\ Jadranska 19, SI-1000 Ljubljana, Slovenia \\ ${ }^{2}$ Rudolf Peierls Centre for Theoretical Physics, Oxford University, \\ Parks Road, Oxford OX1 3PU, United Kingdom
}

(Received 2 November 2020; revised 18 December 2020; accepted 7 April 2021; published 10 May 2021)

\begin{abstract}
We study the time-evolution operator in a family of local quantum circuits with random fields in a fixed direction. We argue that the presence of quantum chaos implies that at large times the time-evolution operator becomes effectively a random matrix in the many-body Hilbert space. To quantify this phenomenon, we compute analytically the squared magnitude of the trace of the evolution operatorthe generalized spectral form factor-and compare it with the prediction of random matrix theory. We show that for the systems under consideration, the generalized spectral form factor can be expressed in terms of dynamical correlation functions of local observables in the infinite temperature state, linking chaotic and ergodic properties of the systems. This also provides a connection between the many-body Thouless time $\tau_{\text {th }}$ - the time at which the generalized spectral form factor starts following the random matrix theory prediction - and the conservation laws of the system. Moreover, we explain different scalings of $\tau_{\text {th }}$ with the system size observed for systems with and without the conservation laws.
\end{abstract}

DOI: 10.1103/PhysRevLett.126.190601

The concept of chaos is very natural in classical systems. Its naive formulation in terms of strong sensitivity of the trajectory to the initial conditions, the "butterfly effect," is so simple and powerful that it has long become an element of the popular culture. During the second half of the 20th century, this concept has been refined from both the physical and mathematical points of view, leading to a complete theory of chaos in classical dynamical systems [1-4] that can be regarded as one of the greatest achievements of mathematical physics.

In the quantum realm, the situation is much less intuitive due to the absence of well-defined trajectories and the linear structure of the unitary evolution. In this context, a key role is played by the spectral correlations of the timeevolution operator. Indeed, as established in a series of seminal works [5-7], systems with a well-defined chaotic classical limit have a spectrum with correlations that coincide with those of an ensemble of random matrices with the same symmetries. The latter property remains well defined also away from the classical limit and has then been taken as a definition of quantum chaos. However, the question of connecting the spectral statistics with more intuitive dynamical properties of the system remained open.

Published by the American Physical Society under the terms of the Creative Commons Attribution 4.0 International license. Further distribution of this work must maintain attribution to the author(s) and the published article's title, journal citation, and DOI.
Over the last decade, the problem of characterizing chaos in quantum systems received renewed interest due to seminal results coming for the study of black holes [8,9] and connecting quantum many-body chaos with the scrambling of quantum information. In turn, this renaissance also produced new discoveries concerning chaos in extended quantum manybody systems on the lattice [10-26] and led to the introduction of useful minimal models like local random unitary circuits [11,27] and dual-unitary circuits [28]. For some of these systems, it has been possible to compute measures of the spectral statistics $[10,12-14,18]$, proving that they indeed follow the predictions of random matrix theory (RMT). Importantly, however, it has been realized that in generic extended systems with local interactions this happens only for energy ranges smaller than a certain scale $E_{\mathrm{th}}$-known as Thouless energy - which bares information on the spatial structure. This energy scale (or the associated Thouless time $\left.\tau_{\mathrm{th}}=\hbar / E_{\mathrm{th}}\right)$ is believed to display different scalings with the system size depending on the conservation laws of the system.

In the recent comeback of quantum chaos, an important role has been played by driven systems, as they furnish a simpler modelization of many interesting dynamical phenomena [23-27]. For these systems, in the generic instance of aperiodic driving, the spectral statistics is not well defined (their time-evolution operator is time dependent), and the chaotic regime has been identified by looking at some features of the quantum many-body dynamics-seeking a quantum many-body analog of the butterfly effect. Some of the most studied features have been the spreading of support of local operators (measured, e.g., by out-of-time-ordered 
correlators [29-31]), the growth of complexity in the classical simulations of the dynamics [32], and the scrambling of quantum information [33]. However, even though all these features are connected to an idea of "dynamical complexity," they provide different information. It is unclear what is the minimal set of these features, if any, that a system has to display to be considered chaotic.

In this Letter, we follow a different route and regard as "chaotic" those driven systems where the time-evolution operator acquires random matrix spectral correlations after a certain initial transient $[34,35]$. This is a direct generalization of the traditional definition of quantum chaos and the transient is naturally interpreted as the Thouless time [35]. We present a family of local quantum circuits with random fields in a fixed direction. In these systems that have no semiclassical limit, the time-dependent spectral correlations can be characterized exactly. In particular, we compute the squared magnitude of the trace of the evolution operator, which we dub the generalized spectral form factor (GSFF), and show that at the leading order in time it is fully specified by the two-point dynamical correlation functions of local operators in the infinite temperature state. This provides an unprecedented direct link between spectral properties and local physics. We use this result to show that the regime of quantum chaos coincides with the ergodic and mixing one (where all dynamical correlations decay in time) and to elucidate the connection between conservation laws and scaling of the Thouless time with the system size.

More specifically, we consider a chain of length $L$ with $2 L$ qubits placed at integer and half-integer indexed sites. Thus, the Hilbert space of the system is $\mathcal{H}=\left(\mathbb{C}^{2}\right)^{\otimes 2 L}$, with dimension $\mathcal{N}=2^{2 L}$. The time evolution is governed by a brickworklike local quantum circuit consisting of unitary matrices (gates) acting on two neighboring spins (with periodic boundary conditions). We consider the case where the gates are different at each space-time point and represent the time evolution as

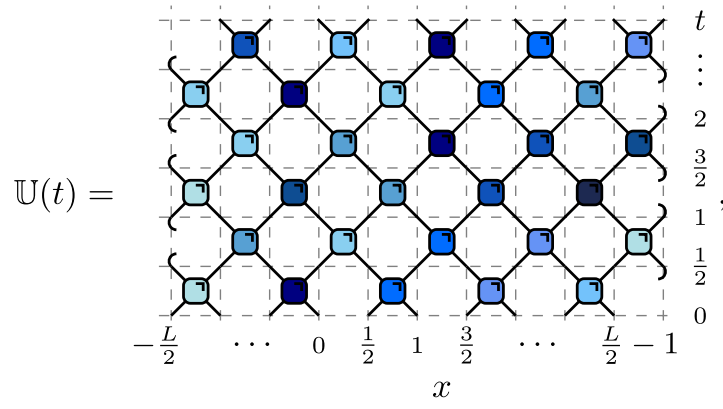

where we depicted two-site gates as

$$
U_{x, \tau}=\sqrt{\widetilde{T}}, \quad x \in \frac{1}{2} \mathbb{Z}_{2 L}, \tau \in \frac{1}{2} \mathbb{Z}_{2 t}, \quad x+\tau \in \mathbb{Z},
$$

and different colors denote different matrices. Note that we adopt the convention of time running upward. We remark that this setting is in fact quite general. It can be thought of as generated by a disordered local Hamiltonian, which changes at each half-integer time due to some external driving. This formulation of quantum evolution is widely used, for instance, in the context of quantum simulators [36].

The main quantity of interest for this Letter is the GSFF defined as

$$
K_{g}(t) \equiv\left\langle\left|\operatorname{tr}_{\text {sector }} \mathbb{U}(t)\right|^{2}\right\rangle, \quad t>0,
$$

where the trace is reduced to a common eigenspace of $\mathbb{U}(t)$ and all its commuting symmetries, and $\langle\cdot\rangle$ denotes an average of some sort (either a moving time average or an average over an ensemble of similar systems). Such an average is necessary because the distribution of $|\operatorname{tr} \mathbb{U}(t)|^{2}$ in an ensemble of systems does not generically become infinitely peaked even in the limit of infinitely many degrees of freedom [37]. From the definition (3), we see that $K_{g}(t)$ with unrestricted trace can be interpreted as the survival probability (or Loschmidt echo) for a random initial state, which is another chaos indicator $[38,39]$.

As mentioned before, here we regard $\mathbb{U}(t)$ as chaotic if there exists a scale $\tau_{\text {th }}$ such that

$$
K_{g}(t) \simeq 1=\mathbb{E}_{\mathrm{CUE}}\left[|\operatorname{tr} U|^{2}\right], \quad \text { for } t \gg \tau_{\mathrm{th}},
$$

where $\simeq$ denotes asymptotic equality in the leading and possibly subleading order in $\tau_{\text {th }} / t$ and, since our system is not time-reversal invariant, we considered the average over the circular unitary ensemble (CUE) [40,41]. Equation (4) allows us to illustrate that expression (3) is very different from the (conventional) spectral form factor considered in periodically driven systems [40-42]. Indeed, here the full time-evolution operator $\mathbb{U}(t)$ is expected to behave like a random matrix at large times, while in the periodically driven case $\mathbb{U}(t)$ behaves as the $t$ th power of a random matrix. This means that $K_{g}(t)$ should be compared with the conventional spectral form factor $K(t)$ at time $t=1$ and that the asymptotic value in Eq. (4) has nothing to do with the asymptotic value $K(t)=\mathcal{N}=2^{2 L}$ at which the conventional spectral form factor relaxes for times larger than the inverse level spacing, namely, for $t>\mathcal{N}$.

In our setting (1), a natural way to produce ensembles of different systems is to introduce noise in the local gate $U_{x, t}$. Since we are interested in generic drivings, we look at timedependent noise and, to avoid any bias, we choose it to be independently distributed in space and time. Specifically, following Ref. [43], we take random gates of the form

$$
U_{x, \tau}=\left(e^{i \phi_{x, \tau} \sigma^{z}} \otimes e^{i \phi_{x+1 / 2, \tau} \sigma^{2}}\right) U,
$$

where $U$ is a fixed $\mathrm{U}(4)$ matrix, $\phi_{x, t}$ are independent random variables uniformly distributed over $[-\pi, \pi]$, and $\otimes$ denotes the tensor product between two neighboring sites. From the physical point of view, the choice (5) describes a homogeneous spin- $1 / 2$ chain where the time evolution is periodic, but each spin is subject to white noise produced by a random magnetic field in the $z$ direction [44]. 
For gates of the form (5), the average $\langle\cdot\rangle$ in Eq. (3) can be implemented locally by placing $\mathbb{U}(t)^{\dagger}$ on top of $\mathbb{U}(t)$ in such a way that each gate lies on top of its conjugate (i.e., "folding" the circuit; see, e.g., Ref. [43]). Specifically, the average projects to a subspace spanned by diagonal operators $\left(|\bigcirc\rangle \equiv|\mathbb{1}\rangle\right.$ and $\left.|\mathbf{O}\rangle \equiv\left|\sigma^{z}\right\rangle\right)$ and allows us to write Eq. (3) as

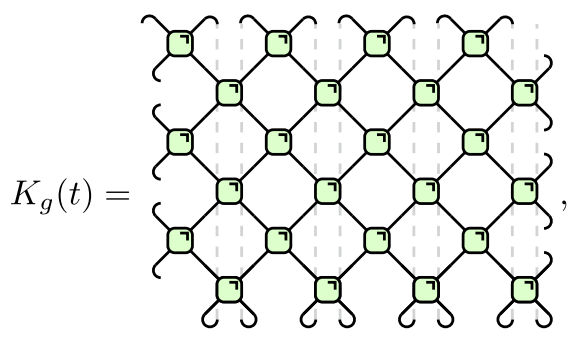

where top and bottom wires at the same position are connected because of the trace. Above we introduced the nonunitary "averaged gate" written in the local basis $\{|\bigcirc\rangle,|\mathbf{O}\rangle\}$ :

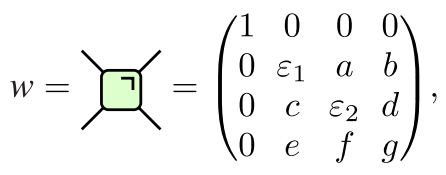

with nine real parameters in $[-1,1]$ depending on the choice of $U$ in Eq. (5) [45]. Remarkably, for any $U, w$ becomes bistochastic after a Hadamard transformation on the single wires [43]. This means that for the choice (5) of noise, $K_{g}(t, L)$ can be interpreted as the state-averaged return probability in a classical stochastic Markov process built as a brickwork circuit with the gate $w$.

Let us now evaluate the first two orders in the asymptotic expansion of Eq. (6) for large times. Conceptually, this will parallel similar derivations carried out in the periodically driven case, in both single-particle [47,48] and many-body $[10,12,13,16,18,35,49]$ contexts. Indeed, even though $K_{g}(t)$ will generically relax to 1 and not to $t$ [cf. Eq. (4)], in both cases the leading correction is exponential, and the relaxation timescale can be interpreted as a Thouless time.

To proceed, we now expand the trace (6) in the computational basis $\left\{\left|e_{i}^{m}\right\rangle\right\}$ where $m=0, \ldots, 2 L$ denotes the particle number (number of quasiparticles 0 ), and $i=1, \ldots,\left(\begin{array}{c}2 L \\ m\end{array}\right)$ labels states in a fixed $m$ sector. Assuming that there are no conserved charges, we have

$K_{g}(t)=\sum_{m=0}^{2 L} K_{g}^{(m)}(t)=1+K_{g}^{(1)}(t)+\cdots+K_{g}^{(2 L)}(t)$,

where we defined in "first quantization notation"

$$
K_{g}^{(m)}(t) \equiv \sum_{\left\{x_{j}\right\}_{j=1}^{m}}^{x_{i}>x_{j}: i>j}\left\langle\boldsymbol{\bullet}_{x_{1}} \ldots \boldsymbol{\bullet}_{x_{m}} \mid \boldsymbol{}_{x_{1}} \ldots \boldsymbol{\bullet}_{x_{m}}(t)\right\rangle_{L}
$$

and used that $K_{g}^{(0)}(t)=1$. We see that $K_{g}^{(m)}(t)$ is expressed as the sum of the averaged autocorrelation functions of the extended operators $\sigma_{x_{1}}^{z} \cdots \sigma_{x_{m}}^{z}$ (with $x_{1}<x_{2} \cdots<x_{m}$, and $x_{j} \in \mathbb{Z}_{2 L} / 2$ ) in finite volume $L$ (cf. Ref. [43]).

Let us now focus on a special family of reduced gates (7): those with either no splittings $(f=e=0)$ or no mergers $(b=d=0)$ and with non-negative weights. For this family of gates, we can invoke the following property (proven in Sec. II of the Supplemental Material [45]):

Property 1. The averaged dynamical correlations $\left\langle\boldsymbol{\bullet}_{x_{1}} \cdots \boldsymbol{\bullet}_{x_{m}} \mid \boldsymbol{\ominus}_{y_{1}} \cdots \boldsymbol{\bullet}_{y_{m}}(t)\right\rangle_{L}$ are bounded from above by $\max \left(1, \frac{g}{\varepsilon_{1} \varepsilon_{2}+a c}\right)^{(m-1) t} \sum_{\sigma \in S_{m}} \prod_{i=1}^{m}\left\langle\boldsymbol{\bullet}_{x_{i}} \mid \boldsymbol{\bullet}_{y_{\sigma(i)}}(t)\right\rangle_{L}$,

where $S_{m}$ is the permutation group of $m$ elements.

Moreover, we also have

Property 2. The two-point functions have the following asymptotic expansion in $t$,

$$
\left\langle\boldsymbol{\odot}_{x} \mid \boldsymbol{\bigotimes}_{y}(t)\right\rangle_{L} \simeq \frac{C_{\eta_{x}, \eta_{y}}}{C_{0,0}+C_{1,1}} \frac{\lambda^{t}}{L}+a_{\eta_{x}}^{2 t} \delta_{t-(x-y) \bmod L},
$$

where $\eta_{x}=2 x \bmod 2, \delta_{0}=1, \delta_{x \neq 0}=0, a_{0}=a, a_{1}=c$,

$$
\lambda=\frac{1}{4}\left((a+c)+\sqrt{4 \varepsilon_{1} \varepsilon_{2}+(a-c)^{2}}\right)^{2},
$$

while $C_{\eta_{x}, \eta_{y}}$ are constant amplitudes $\left(C_{0,0}\right.$ and $C_{1,1}$ are reported in Sec. III of the Supplemental Material [45]).

An instructive way to obtain the expansion (11) is to note that the correlations in finite volume can be written as

$$
\left\langle\boldsymbol{\bullet}_{x} \mid \boldsymbol{\bullet}_{y}(t)\right\rangle_{L}=\sum_{w=-\lfloor t / L\rfloor}^{\lfloor t / L\rfloor}\left\langle\boldsymbol{\bullet}_{x+w L} \mid \boldsymbol{\bullet}_{y}(t)\right\rangle_{\infty},
$$

where $\left\langle\boldsymbol{\bullet}_{x} \mid \boldsymbol{\Theta}_{y}(t)\right\rangle_{\infty}$ are the infinite volume correlations known exactly from Ref. [43]. This form follows from the observation that for no splittings (mergers) the only contributions to the correlation come from continuous paths (the skeleton diagrams [43]) connecting the end points, and wrapping around the cylindrical world sheet along the space direction an arbitrary number of times. The maximal number of wrappings is restricted by the maximal speed of propagation. Then, Eq. (11) follows directly from plugging in the asymptotic form

$$
\left\langle\boldsymbol{\bullet}_{x} \mid \boldsymbol{\Theta}_{y}(t)\right\rangle_{\infty} \simeq \delta_{t-(x-y)} a_{\eta_{x}}^{2 t}+\frac{\lambda^{t} C_{\eta_{x}, \eta_{y}}}{\sqrt{t}} e^{-\frac{(x-y-\bar{t} t)^{2}}{4 D t}}
$$

[where the diffusion constant is given by $D=\left[4 \pi\left(C_{0,0}+\right.\right.$ $\left.\left.C_{1,1}\right)^{2}\right]^{-1}$ and the fluid velocity $\bar{\zeta}$ is defined in Sec. III of the Supplemental Material [45]], and turning the sum over $w L / t$ into an integral for $t \gg L$. Alternatively, Eq. (11) can also be derived by diagonalizing an effective Markov operator; see Sec. IV of the Supplemental Material [45]. 
Using the asymptotic form (11) for two-point correlations and Property 1, we find (see Sec. V of the Supplemental Material [45])

$$
\sum_{m=2}^{2 L} K_{g}^{(m)}(t)<C L^{2} \lambda^{2 t} \max \left(1, \frac{g^{t}}{\left(\varepsilon_{1} \varepsilon_{2}+a c\right)^{t}}\right) .
$$

This leads us to our first main result: For large times and $\lambda \max \left[1, g /\left(\varepsilon_{1} \varepsilon_{2}+a c\right)\right]<1$, the GSFF is fully determined by correlation functions of local observables

$$
K_{g}(t) \simeq 1+K_{g}^{(1)}(t) \simeq 1+\lambda^{t}+\left(a^{2 t}+c^{2 t}\right) L \delta_{t \bmod L} .
$$

In particular, since $\lambda>\max \left(a^{2}, c^{2}\right)$, we find

$$
K_{g}(t) \simeq 1+e^{-t / \tau_{\text {th }}}, \quad \tau_{\text {th }}^{-1}=-\log \lambda .
$$

Note that in this case, $\tau_{\text {th }}$ is the exponent governing the decay of two-point correlations in infinite volume. Note also that there is no $L$ dependence in $\tau_{\text {th }}$, in contrast to $\log L$ dependence found in several examples of extended systems; see, e.g., Refs. [10,12,35].

Equation (16) shows excellent agreement with the exact numerical evaluation of $K_{g}(t)$; see Fig. 1 for a representative example. Moreover, our numerical observations suggest that the bound (15) is too conservative, and Eq. (16) holds whenever $\lambda<1$, namely, whenever the averaged two-point correlations decay exponentially.

When some of the gate's parameters (7) are negative, the Gaussian asymptotic form (14) is not valid. We calculate $K_{g}^{(1)}(t) \simeq \lambda^{t}$ by diagonalizing an effective Markov operator; see Sec. IV of the Supplemental Material [45] [ $\lambda$ can be different from the one in Eq. (12)]. Moreover, we again bound the other contributions as in Eq. (15) (with a minor modification; see Sec. V of the Supplemental Material [45]).

Let us now consider a special case for which Eq. (15) does not provide a useful bound (because $\lambda=1$ ). Namely, the case of averaged gates with a conservation law. This situation has been extensively studied in the recent literature $[13,35,50,51]$ and can be realized in our setting by considering a gate $U$ [and hence, $U_{x, \tau}$ in Eq. (5)] that conserves the magnetization in the $z$ direction. This leads to the following averaged gate [45]:

$w_{U(1)}=\left(\begin{array}{cccc}1 & 0 & 0 & 0 \\ 0 & \cos ^{2} 2 J & \sin ^{2} 2 J & 0 \\ 0 & \sin ^{2} 2 J & \cos ^{2} 2 J & 0 \\ 0 & 0 & 0 & 1\end{array}\right), \quad J \in[0, \pi / 4]$.

Note that the time-evolution operator generated by this gate is integrable: It is an example of the Floquet XXX model at a nonunitary point [52]. Interestingly, a similar Floquet XXX model was obtained in Ref. [13] after averaging a U(1)-symmetric Floquet-Haar random circuit. Finally, we remark that a similar reduced gate for driven systems has been studied in Ref. [35].

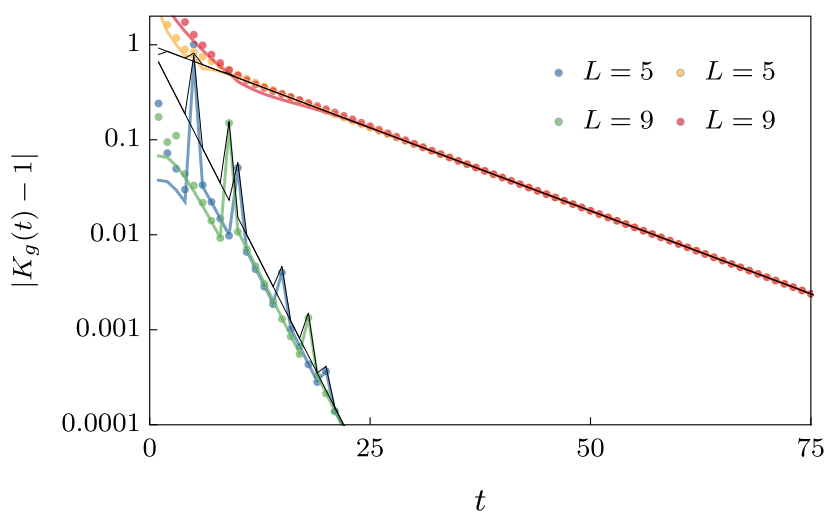

FIG. 1. Deviations of GSFF $K_{g}(t)$ from the RMT prediction for two different gates with no splittings, $b=d=0$. Symbols denote the exact numerical results for up to 18 sites $(L=9)$. Solid color line depicts $K_{g}^{(1)}(t)$ computed according to Eqs. (9) and (13). Notice that the $\tau_{\text {th }}$ does not scale with $L$. The solid black lines show the asymptotic from Eq. (16). We wrote the gate's parameters in Table SM-1 of Sec. VII of the Supplemental Material [45].

Since the magnetization is conserved, the trace in Eq. (3) is reduced to a single magnetization sector. This means that instead of $K_{g}(t)$ in Eq. (8), we should consider a single term $K_{g}^{(m)}(t)$ with fixed $m=0,1, \ldots, 2 L$. Moreover, we observe that, apart from the two trivial sectors $m=0$ and $m=2 L$ where the GSFF is 1 , all $K_{g}^{(m)}(t)$ decay to 1 with the same exponent; see Fig. 2. This can be understood directly from the Bethe-ansatz solution (see, e.g., the Supplemental Material of Ref. [13]). Indeed, by looking at the finite volume eigenstates, one finds that the lowest excitations (those with the eigenvalue of the Markov operator which is the closest to 1) are one-magnon excitations (as opposed to bound states or scattering states of many magnons). Since the one-magnon states are the highest-weight states of the representation of $\mathrm{SU}(2)$ with $S_{z}=L-1$, their descendants (obtained by multiple applications of the lowering operators $S^{-}$) appear in all sectors $m=1, \ldots, 2 L-1$. Therefore, all sectors have

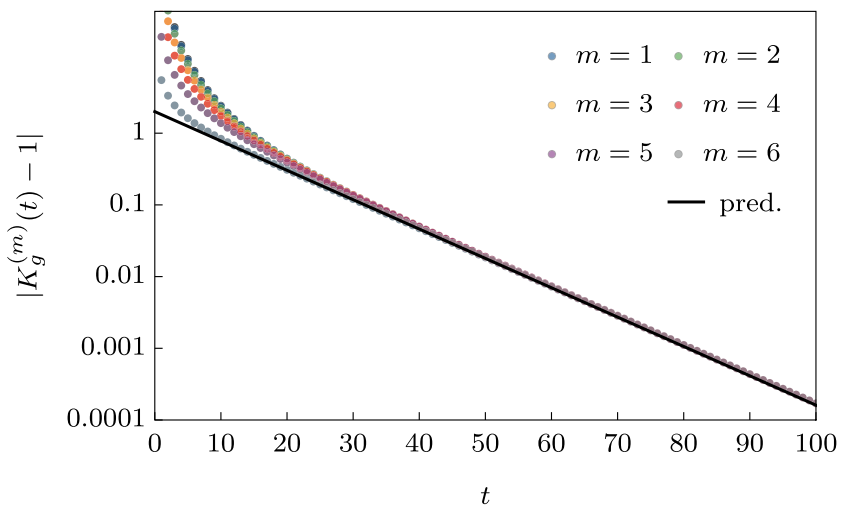

FIG. 2. Deviations of the GSFF $\left|K_{g}^{(m)}(t)-1\right|$ with conservation laws. We show the results for different magnetization sectors at $L=7$ (14 sites) and $J=0.3$. The black line is the prediction (21). 
the same Thouless time, which can be deduced from the $m=1$ sector.

For large times, the averaged two-point function for $m=1$ takes a simple diffusive form

$$
\left\langle\boldsymbol{O}_{x} \mid \boldsymbol{\Theta}_{0}(t)\right\rangle_{\infty} \simeq \frac{1}{2 \sqrt{4 \pi t D}} e^{-\frac{x^{2}}{4 D t}},
$$

where $D=\left(\tan ^{2} 2 J\right) / 4$ is the diffusion constant, and we neglected exponentially small corrections with $L$ independent exponents because we expect an $L$-dependent Thouless time. Using again Eq. (13), we have

$$
K_{g}^{(1)}(t) \simeq \frac{1}{2 \sqrt{4 \pi t D}} \sum_{w=-\lfloor t / L\rfloor}^{\lfloor t / L\rfloor} e^{-\frac{w^{2} L^{2}}{4 D t}} .
$$

Extending the summation to $\pm \infty$ [53] and utilizing the Poisson summation formula, we get

$K_{g}^{(1)}(t) \simeq \sum_{n=-\infty}^{\infty} e^{-\frac{4 \pi^{2} D t n^{2}}{L^{2}}} \simeq 1+2 e^{-t / \tau_{\text {th }}}, \quad \tau_{\text {th }}=\frac{L^{2}}{4 \pi^{2} D}$.

Note that the Thouless time depends on $L^{2} / D$, in agreement with previous observations in chaotic systems with diffusive conservation laws in both single-particle $[47,48]$ and manybody $[13,16,18,35,49]$ contexts. Our derivation gives a straightforward illustration of the origin of this scaling.

Another interesting limiting case is when, in addition to no splittings (or merges), at least one of $\varepsilon_{1}$ and $\varepsilon_{2}$ vanishes (note that $\varepsilon_{1}=\varepsilon_{2}=0$ if and only if the gate $U$ is dual unitary $[28,43])$. In this case, $K_{g}(t)=1+\left(a^{2 t}+c^{2 t}\right) L \delta_{t \bmod L}+\ldots$, and the GSFF admits a closed-form expression (see Sec. VI of the Supplemental Material [45]). The model is chaotic when all $a, c, g$ differ from \pm 1 . In contrast, if the above conditions do not hold, $K_{g}(t)$ with unrestricted trace does not decay to the RMT result. This signals new commuting symmetries and possibly nonchaotic behavior. For instance, for $a=c=g=1$ (corresponding to the SWAP gate) and unrestricted trace we find $\left.K_{g}(t)\right|_{\mathrm{SWAP}}=4^{\mathrm{GCD}(t, L)}$. Here $\operatorname{GCD}(t, L)$ is the greatest common divisor of $L$ and $t$. This result is manifestly larger than the RMT result.

In the general case, when both mergers and splittings are allowed, there is a phase transition in the decay exponent of infinite volume correlations [43]. In particular, there is a region in parameter space [see Eq. (41) in Ref. [43]] where the decay of quasiparticles is still governed by $\lambda$ in Eq. (12), while for parameters out of this region the exponent changes. Moreover, all $K_{g}^{(m)}(t)$ will decay with the same exponent [since the number of particles can change during the time evolution, all $K_{g}^{(m)}(t)$ contain the slow-decaying configurations]. However, this means that the decay exponent can again be determined from two-point functions of local operators and that $\tau_{\text {th }}=-1 / \log \lambda_{\max }$, where $\lambda_{\text {max }}=\lim _{t \rightarrow \infty}\left(\max _{x}\left\langle\boldsymbol{\bigcirc}_{x} \mid \boldsymbol{\bigcirc}_{0}(t)\right\rangle_{\infty}\right)^{1 / t}$. This is in agreement with our numerical experiments, as shown Fig. 3 for a representative example.

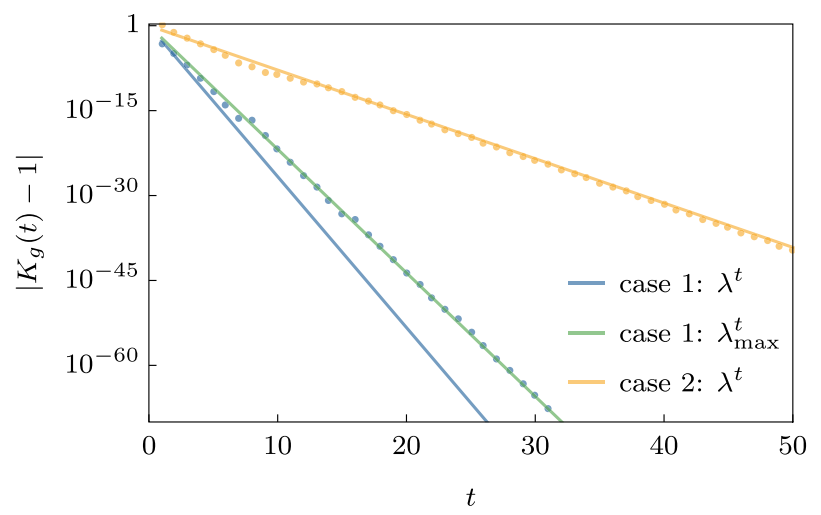

FIG. 3. Symbols show the numerical results for deviations of the spectral form factor $|K(t)-1|$ with allowed splittings and mergers. Solid lines show the decay of the skeleton correlation functions $\lambda^{t}$ from Eq. (12) and the true decay of the two-point correlation function $\lambda_{\max }^{t}$. In case 2 , the two-point correlation function is well described by the skeleton contributions (see Ref. [43] for when this holds). In contrast, case 1 exhibits a slower decay of the deviations than given by (12), agreeing with the slower decay of the correlation functions. We obtained $\lambda_{\max }$ from direct numerical evaluation of the two-point correlation functions in infinite volume. The data shown are for the last two gates in Table SM-1 of Sec. VII of the Supplemental Material [45], $L=8$.

In conclusion, we studied the GSFF in a class of local quantum circuits with random fields, expressing it in terms of (averaged) dynamical correlations of local observables. By means of this correspondence, we showed that in the regime where the correlations decay exponentially in time (known as ergodic and mixing in ergodicity theory), the GSFF approaches the prediction of random matrix theory over the same timescale. Moreover, we proved that the GSFF approaches the prediction of random matrix theory also in the presence of a conservation law if the correlations take a diffusive form. In this case, the timescale is proportional to the system size squared divided by the diffusion constant. Finally, we showed that when the correlations do not decay, the GSFF does not approach the random matrix theory prediction. The correspondence between quantum chaotic and quantum ergodic and mixing regimes is expected on general grounds [39,54-56] and provides an intuitive understanding of quantum chaos. Our results in a specific setting provide a rigorous proof of such a correspondence, and pave the way for its quantitative understanding in more general settings. Moreover, interpreting the U(1)-noiseaveraged GSFF as a state-averaged return probability for a general bistochastic brickwork Markov circuit provides an analogous correspondence in classical stochastic systems.

The work is supported by the EU Horizon 2020 program through the ERC Advanced Grant OMNES No. 694544, and by the Slovenian Research Agency under the Program 
No. P1-0402. B. B. was also supported by the Royal Society through the University Research Fellowship No. 201102.

[1] V. I. Arnold and A. Avez, Ergodic Problems of Classical Mechanics, Reprint ed. (Addison-Wesley, Reading, MA, 1989).

[2] V.I. Arnold, Geometrical Methods in the Theory of Ordinary Differential Equations (Springer, New York, 1988).

[3] E. Ott, Chaos in Dynamical Systems, 2nd ed. (Cambridge University Press, Cambridge, England, 2012).

[4] I. P. Cornfeld, S. V. Fomin, and Y. G. Sinai, Ergodic Theory (Springer, New York, 2012).

[5] G. Casati, F. Valz-Gris, and I. Guarneri, On the connection between quantization of nonintegrable systems and statistical theory of spectra, Lett. Nuovo Cimento 28, 279 (1980).

[6] M. V. Berry, Quantizing a classically ergodic system: Sinai's billiard and the KKR method, Ann. Phys. (N.Y.) 131, 163 (1981).

[7] O. Bohigas, M. J. Giannoni, and C. Schmit, Characterization of Chaotic Quantum Spectra and Universality of Level Fluctuation Laws, Phys. Rev. Lett. 52, 1 (1984).

[8] P. Hayden and J. Preskill, Black holes as mirrors: Quantum information in random subsystems, J. High Energy Phys. 09 (2007) 120.

[9] Y. Sekino and L. Susskind, Fast scramblers, J. High Energy Phys. 10 (2008) 065.

[10] P. Kos, M. Ljubotina, and T. Prosen, Many-Body Quantum Chaos: Analytic Connection to Random Matrix Theory, Phys. Rev. X 8, 021062 (2018).

[11] A. Chan, A. De Luca, and J. T. Chalker, Solution of a Minimal Model for Many-Body Quantum Chaos, Phys. Rev. X 8, 041019 (2018).

[12] A. Chan, A. De Luca, and J. T. Chalker, Spectral Statistics in Spatially Extended Chaotic Quantum Many-Body Systems, Phys. Rev. Lett. 121, 060601 (2018).

[13] A. J. Friedman, A. Chan, A. De Luca, and J. T. Chalker, Spectral Statistics and Many-Body Quantum Chaos with Conserved Charge, Phys. Rev. Lett. 123, 210603 (2019).

[14] B. Bertini, P. Kos, and T. Prosen, Exact Spectral Form Factor in a Minimal Model of Many-Body Quantum Chaos, Phys. Rev. Lett. 121, 264101 (2018).

[15] S. J. Garratt and J. T. Chalker, Many-Body Quantum Chaos and the Local Pairing of Feynman Histories, arXiv: 2008.01697 [Phys. Rev. X (to be published)].

[16] S. Moudgalya, A. Prem, D. A. Huse, and A. Chan, Spectral statistics in constrained many-body quantum chaotic systems, arXiv:2009.11863.

[17] A. Keselman, L. Nie, and E. Berg, Scrambling and Lyapunov exponent in unitary networks with tunable interactions, Phys. Rev. B 103, L121111 (2021).

[18] D. Roy and T. Prosen, Random matrix spectral form factor in kicked interacting fermionic chains, Phys. Rev. E 102, 060202(R) (2020).

[19] B. Bertini, P. Kos, and T. Prosen, Operator entanglement in local quantum circuits I: Chaotic dual-unitary circuits, SciPost Phys. 8, 067 (2020).
[20] B. Bertini, P. Kos, and T. Prosen, Operator entanglement in local quantum circuits II: Solitons in chains of qubits, SciPost Phys. 8, 068 (2020).

[21] P. W. Claeys and A. Lamacraft, Maximum velocity quantum circuits, Phys. Rev. Research 2, 033032 (2020).

[22] V. Alba, J. Dubail, and M. Medenjak, Operator Entanglement in Interacting Integrable Quantum Systems: The Case of the Rule 54 Chain, Phys. Rev. Lett. 122, 250603 (2019).

[23] C. Jonay, D. A. Huse, and A. Nahum, Coarse-grained dynamics of operator and state entanglement, arXiv:1803.00089.

[24] B. Bertini and L. Piroli, Scrambling in random unitary circuits: Exact results, Phys. Rev. B 102, 064305 (2020).

[25] A. Nahum, S. Vijay, and J. Haah, Operator Spreading in Random Unitary Circuits, Phys. Rev. X 8, 021014 (2018).

[26] C. W. von Keyserlingk, T. Rakovszky, F. Pollmann, and S. L. Sondhi, Operator Hydrodynamics, OTOCs, and Entanglement Growth in Systems without Conservation Laws, Phys. Rev. X 8, 021013 (2018).

[27] A. Nahum, J. Ruhman, S. Vijay, and J. Haah, Quantum Entanglement Growth under Random Unitary Dynamics, Phys. Rev. X 7, 031016 (2017).

[28] B. Bertini, P. Kos, and T. Prosen, Exact Correlation Functions for Dual-Unitary Lattice Models in $1+1$ Dimensions, Phys. Rev. Lett. 123, 210601 (2019).

[29] S. H. Shenker and D. Stanford, Black holes and the butterfly effect, J. High Energy Phys. 03 (2014) 067.

[30] D. A. Roberts, D. Stanford, and L. Susskind, Localized shocks, J. High Energy Phys. 03 (2015) 51.

[31] J. Maldacena, S. H. Shenker, and D. Stanford, A bound on chaos, J. High Energy Phys. 08 (2016) 106.

[32] T. Prosen and M. Žnidarič, Is the efficiency of classical simulations of quantum dynamics related to integrability?, Phys. Rev. E 75, 015202(R) (2007).

[33] P. Hosur, X.-L. Qi, D. A. Roberts, and B. Yoshida, Chaos in quantum channels, J. High Energy Phys. 02 (2016) 004.

[34] P. Saad, S. H. Shenker, and D. Stanford, A semiclassical ramp in SYK and in gravity, arXiv:1806.06840.

[35] H. Gharibyan, M. Hanada, S. H. Shenker, and M. Tezuka, Onset of random matrix behavior in scrambling systems, J. High Energy Phys. 07 (2018) 124.

[36] F. Arute, K. Arya, R. Babbush et al., Quantum supremacy using a programmable superconducting processor, Nature (London) 574, 505 (2019).

[37] R. Prange, The Spectral Form Factor Is Not Self-Averaging, Phys. Rev. Lett. 78, 2280 (1997).

[38] E. J. Torres-Herrera and L. F. Santos, Dynamical manifestations of quantum chaos: correlation hole and bulge, Phil. Trans. R. Soc. A 375, 20160434 (2017).

[39] T. Gorin, T. Prosen, T. H. Seligman, and M. Žnidarič, Dynamics of Loschmidt echoes and fidelity decay, Phys. Rep. 435, 33 (2006).

[40] F. Haake, Quantum Signatures of Chaos, 2nd ed. (Springer, New York, 2001).

[41] M. L. Mehta, Random Matrices and the Statistical Theory of Spectra, 2nd ed. (Academic, New York, 1991).

[42] H. Kunz, The probability distribution of the spectral form factor in random matrix theory, J. Phys. A 32, 2171 (1999). 
[43] P. Kos, B. Bertini, and T. Prosen, Correlations in Perturbed Dual-Unitary Circuits: Efficient Path-Integral Formula, Phys. Rev. X 11, 011022 (2021).

[44] A similar setting for continuous time has recently been considered in Ref. [57].

[45] See Supplemental Material at http://link.aps.org/ supplemental/10.1103/PhysRevLett.126.190601 for some useful complementary information, which includes Ref. [46].

[46] ITensor Library (version 3), http://itensor.org.

[47] O. L. Al'tshuler and B. I. Shklovskii, Repulsion of energy levels and conductivity of small metal samples, J. Exp. Theor. Phys. 64, 127 (1986), http://www.jetp.ac.ru/cgi-bin/ e/index/e/64/1/p127?a=list.

[48] N. Argaman, Y. Imry, and U. Smilansky, Semiclassical analysis of spectral correlations in mesoscopic systems, Phys. Rev. B 47, 4440 (1993).

[49] J. Šuntajs, J. Bonča, T. Prosen, and L. Vidmar, Quantum chaos challenges many-body localization, Phys. Rev. E 102, 062144 (2020).

[50] V. Khemani, A. Vishwanath, and D. A. Huse, Operator Spreading and the Emergence of Dissipative Hydrodynamics under Unitary Evolution with Conservation Laws, Phys. Rev. X 8, 031057 (2018).

[51] T. Rakovszky, F. Pollmann, and C. W. von Keyserlingk, Diffusive Hydrodynamics of Out-of-Time-Ordered Correlators with Charge Conservation, Phys. Rev. X 8, 031058 (2018).

[52] M. Vanicat, L. Zadnik, and T. Prosen, Integrable Trotterization: Local Conservation Laws and Boundary Driving, Phys. Rev. Lett. 121, 030606 (2018).

[53] It can be done up to exponential corrections with $L$-independent characteristic time.

[54] T. Prosen, Chaos and complexity of quantum motion, J. Phys. A 40, 7881 (2007).

[55] M. Degli Esposti, S. Graffi, and S. Isola, Classical limit of the quantized hyperbolic toral automorphisms, Commun. Math. Phys. 167, 471 (1995).

[56] Quantum Chaos between Order and Disorder, edited by G. Casati and B. V. Chirikov (Cambridge University Press, Cambridge, England, 1995).

[57] J. Ren, Q. Li, W. Li, Z. Cai, and X. Wang, Noise-Driven Universal Dynamics towards an Infinite Temperature State, Phys. Rev. Lett. 124, 130602 (2020). 